\title{
Metabolic syndrome resolution by Roux-en-Y gastric bypass in a real world: a case control study
}

\author{
Helena Schmid ${ }^{1 *}$, Claudio Fernando Goelzer Neto ${ }^{2}$, lisiane Stefani Dias ${ }^{3}$, Antonio Carlos Weston ${ }^{4}$, Marília Bittencourt Espíndola ${ }^{5}$, \\ Sergio Ricardo Pioner ${ }^{5}$, Luiz Alberto De Carli ${ }^{6}$ \\ ${ }^{1} \mathrm{MD}$, PhD in Endocrinology, Full professor at Universidade Federal do Rio Grande do Sul (UFRGS)/Hospital das Clínicas de Porto Alegre and Santa Casa de Porto Alegre, RS, Brazil \\ ${ }^{2} \mathrm{MSC}$, Graduate student in Health Sciences at Universidade Federal de Ciências da Saúde de Porto Alegre (UFCSPA), Porto Alegre, RS, Brazil \\ ${ }^{3} \mathrm{MSc}$, Graduate student in Gynecology and Obstetrics (PPGGO - UFRGS), Porto Alegre, RS, Brazil \\ ${ }^{4} \mathrm{MD}$, PhD in Surgery, Surgeon at Santa Casa de Porto Alegre, Porto Alegre, RS, Brazil \\ ${ }^{5} \mathrm{MD}, \mathrm{MSc}$ in Surgery, Surgeon at Santa Casa de Porto Alegre, RS, Brazil \\ ${ }^{6} \mathrm{MD}$ - Surgeon at Santa Casa de Porto Alegre, RS, Brazil
}

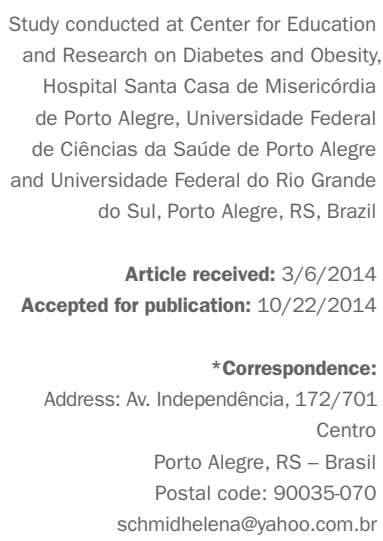

Study conducted at Center for Education and Research on Diabetes and Obesity, Hospital Santa Casa de Misericórdia de Porto Alegre, Universidade Federal de Ciências da Saúde de Porto Alegre and Universidade Federal do Rio Grande do Sul, Porto Alegre, RS, Brazil

Article received: $3 / 6 / 2014$ Accepted for publication: 10/22/2014

*Correspondence: Address: Av. Independência, 172/701 Centro Porto Alegre, RS - Brasi Postal code: $90035-070$ schmidhelena@yahoo.com.br

http://dx.doi.org/10.1590/1806-9282.61.02.161 Conflict of interest: none

\section{SUMmary}

Objectives: the aim of this study was to evaluate the efficacy of Roux-en-Y Gastric Bypass (RYGB), compared with nonsurgical treatment (NS group), as an instrument for inducing remission of co-morbidities related to Metabolic Syndrome (MetS) in patients with Obesity, grades 2 and $3(\mathrm{Ob} 2,3)$.

Methods: two hundred and fifty eight $\mathrm{Ob} 2,3$ patients were selected in a retrospective analysis and included in a case control study. MetS was defined as described by the International Diabetes Federation. One hundred and twenty-nine of these patients underwent RYGB (S group), and 129 were assessed as an NS group.

Results: at baseline, $S$ and NS groups did not differ in BMI, age, female sex and prevalence of MetS ( $p>0.05)$. For the $S$ group the outcomes were a reduction in BMI of $38.1 \%$ ( $\mathrm{p}<0.001)$, waist circumference of $28.6 \%$ ( $\mathrm{p}<0.001)$, fasting plasma glucose of $10.5 \%(\mathrm{p}<0.001)$, serum LDL-cholesterol of $21.9 \%(\mathrm{p}<0.001)$ and of $85 \%$ in the number of patients with MetS $(\mathrm{p}<0.001)$. For the NS group, only a decrease of $4.12 \%(\mathrm{p}=0.047)$ in triglyceride levels and of $5.9 \%(\mathrm{p}=0.031)$ in Diastolic Blood Pressure was observed. In the NS group, $98.6 \%$ of the patients continued to have MetS. The number needed to treat (NNT) with surgery to resolve one case of MetS was 1.2 (CI 95\%: 1.1 - 1.4).

Conclusion: in the real world, in the South of Brazil, compared with NS treatment and after 1 year of observation, RYGB is highly effective for decreasing the prevalence of MetS.

Keywords: metabolic syndrome X, obesity, abdominal obesity, hypertension, hyperglycemia, hyperlipidemias.

\section{INTRODUCTION}

Although it has been well established in the literature that severe obesity is related to Metabolic Syndrome (MetS) and both, obesity and MetS, are associated with increased morbidity and mortality ${ }^{1}$ and a high risk of developing cardiovascular disease, ${ }^{2}$ the best treatment for obese patients with MetS is still being debated. Diet, exercise, and the anti-obesity medications are usually not very effective in inducing and maintaining long-term weight loss, especially for obesity grades 2 and 3.3-5 Because of that, other types of therapeutic tools, such as bariatric surgery, are receiving more attention for evaluation in clinical trials. ${ }^{1}$
It is known, however, that the effectiveness of bariatric surgery, depends on several factors such as type of surgery, availability of skilled surgeons, pre- and post-operative care offered, education about nutritional habits and physical activities of patients and also, the patient's capacity to understand the recommendations. In Brazil, there is not enough information about the real life effects of these surgeries, when many surgeons operate and the care that is offered to patients is similar to the one offered to patients under clinical treatment. Moreover, surgical treatment (ST) has not been recommended for most patients, especially for diabetic patients whose physicians fear surgery-related complications. 
Of the surgical techniques available, the Roux-en-Y Gastric Bypass (RYGB) is the most widely used technique, and the laparoscopic RYGB is considered, by many surgeons, as the gold standard for ST of severe obesity (SO), with relatively low morbidity and mortality. ${ }^{5}$

In 2010, Hofsø et al. ${ }^{4}$ published a one-year trial, comparing RYGB with a very intensive lifestyle intervention in SO patients, and showed 8 and $30 \%$ of weight loss respectively for the clinical group and surgery group. This effect occurred with the use of a cognitive approach in which each patient was motivated to increase physical activities and to change eating habits at a center where the patients stayed for either 1 or 4 weeks, and had contact with the team that was treating them at least once every 2 weeks. It was surprising that, even with this very intensive clinical intervention, the RYGP group had greater improvement compared to the life style intervention group.

In an observational study with 949 patients undergoing RYGB, between 2005 and 2010, weight loss (WL) was strongly associated with glycemic control improvement, blood level of LDL-cholesterol decrease, HDL-cholesterol increase, lower prevalence of hypertriglyceridemia, and reduction of $20 \%$ in the prevalence of high blood pressure. ${ }^{7}$

Taking this and other studies into account, Kaul and Sharma ${ }^{5}$ recommend that SO patients with MetS and associated co-morbidities should be promptly referred for consideration of treatment with bariatric surgery.

Other than the findings and recommendations reported above, we were not able to find any reports of studies that specifically evaluated the evolution of parameters involving MetS in SO patients submitted to clinical and surgical treatments in real life Brazil, other than the findings and recommendations reported above. If MetS could be reversed (resolved), it is expected that its cardiovascular outcomes should also decrease. Since RYGB has a mortality of at least $0.5 \%,{ }^{8}$ it is necessary to compare its results with what can be achieved without submitting the patients to the surgical procedure.

The aim of this study was to evaluate the efficacy of RYGB in resolving MetS and in improving other co-morbidities related to $\mathrm{SO}$ compared to no surgical treatment after one year of follow-up in a Brazilian Multidisciplinary Center, where the mortality for this procedure is the same as that reported in literature ( $0.5 \%)$. SO patients frequently complain of arthralgia related to increased impact of the weight upon the joints, snoring, which is related mainly to the obstruction of the upper airways caused by excess of adipose tissue, and often have high blood pressure; ${ }^{5}$ thus, we also evaluated these parameters in our patients. Data on serum total proteins, albumin, vitamin B12 and iron deficiency anemia were also obtained be- cause RYGB is considered a restrictive and malabsorptive procedure and the patients, in order to prevent anemia, hypoalbuminemia and vitamin B12 deficiency, usually need to receive supplementation with proteins and vitamins after the procedure. ${ }^{7}$

\section{Methods}

\section{Sample population and data collection}

We performed a retrospective cohort study of consecutive patients referred for RYGB that were identified using the records of patients treated for grades 2 and 3 obesity at the Center for Treatment of Obesity (CTO), Santa Rita Hospital, which belongs to the Hospital Santa Casa de Misericórdia de Porto Alegre, state of Rio Grande do Sul, Brazil, between January 1, 2008, and December 31, 2009. These patients were considered as the group of surgical cases (S group). As controls (NS group), we selected patients treated medically (non surgical) in the same hospital and during the same period as the cases. Of the 281 patients that were selected for the S group, 152 were excluded from our sample due to incomplete data (38) or follow-up registered in medical records of less than 3 months after surgery (114). Demographic data on these 152 patients who were excluded did not differ statistically from the enrolled patients. Thus, our S group consisted of 129 patients who received the surgical treatment (ST). The NS patients were referred for clinical treatment (NST) of obesity, because they did not want to undergo surgery and in a few cases $(n=12)$, because they had surgical contraindications. These patients received medical recommendations for changing lifestyle (diet and exercise) in visits that occurred every 3 months (on average) with the nutritionist, endocrinologist, psychologist and psychiatrist. Thus, our NS group consisted of $129 \mathrm{pa}-$ tients followed at the same hospital and time, and treated by the same team of the ST group. For the final formation of the groups, the controls were paired to cases by age, sex, body mass index (BMI) and follow-up time in the outpatient clinics of the hospital. A total of $258 \mathrm{pa}-$ tients were evaluated in our study.

The variables analyzed before and after the interventions were: age, sex, height, weight, BMI, waist circumference, systolic and diastolic blood pressure, mean arterial pressure, history of type 2 diabetes, history of systemic hypertension, history of dyslipidemia, arthralgia, symptoms of snoring, number of medications used and presence of MetS according to the International Diabetes Federation. ${ }^{9}$ Results of 12 hours fasting blood, glucose, total cholesterol, HDL cholesterol, LDL cholesterol, and triglycerides were also considered. The variables collected and analyzed only after surgery and clinical treatment were total serum 
protein, serum albumin, serum vitamin B12, serum iron, serum ferritin, hemoglobin and hematocrit.

The Hospital Santa Casa's CTO is a multidisciplinary team specialized in the treatment of SO. This team consists of professionals working in the fields of clinical medicine, general surgery, endocrinology, gastroenterology, nutrition, psychiatry and psychology. In their first evaluation in the division, if grade 2 and 3 obese patients (SO) had prior attempts to lose weight for at least 2 years, they were asked about the possibility to undergo a surgery as the treatment. Treatment type, surgical or clinical, was then defined. Besides the history of failed weight loss with clinical treatment, surgery was indicated if the patient had: $\mathrm{BMI} \geq 40 \mathrm{Kg} / \mathrm{m}^{2}$ or $\mathrm{BMI} \geq 35 \mathrm{Kg} / \mathrm{m}^{2}$ with co-morbidities, absence of other clinically severe disease identified by abnormalities in the exams listed below, absence of severe psychopathology, absence of history of using illicit drugs and alcoholism, mental conditions that could make the patient misunderstand explanations about the implications of surgery, and age between 18-65 years. Patients with psychosis, cancer and serious infectious diseases did not undergo surgery, being referred for a clinical program to lose weight. They were not included as subjects in this study.

Patients referred for RYGB were given appointments with a multidisciplinary team including nutritionist, psychologist, endocrinologist and general practice doctor. Bariatric surgeries were performed only if all the professionals of the team who saw the patient gave a written agreement. After the patient signed the informed consent form, the surgery was scheduled. In the pre-surgery period, all patients underwent laboratory evaluation (blood count, fasting plasma glucose, alanine aminotransferase (ALT), aspartate aminotransferase (AST), alkaline phosphatase, bilirubin, serum iron, ferritin, vitamin B12, total serum proteins, albumin, calcium, phosphorus, magnesium, zinc, urea, creatinine, total cholesterol, HDL-cholesterol, LDLcholesterol, triglycerides and urine exam), thoracic X-ray, high digestive endoscopy, abdominal ultrasound, electrocardiogram and other exams, if necessary, in order to determine the presence of cancer or infectious diseases.

Almost all the laboratory tests were also performed in the post-surgery period, at visits every 3 months (on average). After ST, all the patients received a prescription for diet supplementation with a complex of vitamins and minerals (Centrum $\left.{ }^{\circledR}\right)$.

NS was another modality of treatment for SO offered by the CTO, based on encouraging a change in lifestyle (diet and exercise) in consultations with nutritionist, endocrinologist, psychologist and psychiatrist every 12 weeks (on average).

\section{Definitions}

Time of RYGB and time of nutrition consultation were considered as the baseline time for the $\mathrm{S}$ and NS groups, respectively. We defined our baseline variables based on information in the medical records at baseline time or earlier; follow-up variables were based on information at time of last follow-up evaluation present in the medical records. All patients in our study had a minimum follow-up of at least 3 months. The average follow-up time of patients in our study was 12 months for $S$ and NS groups. The variables of the study, collected for both groups, before and after their respective treatments were: age, sex, height, weight, BMI, waist circumference (WC), systolic blood pressure (SBP), diastolic blood pressure (DBP), mean blood pressure (MBP), fasting plasma glucose (f-glu), total cholesterol, HDL-cholesterol (HDL-chol), LDL-cholesterol (LDL-chol), triglycerides, history of T2DM, history of hypertension, history of dyslipidemia, symptoms of arthralgia, symptoms of snoring and number of drugs used. For the assessment of clinical nutritional complications after treatments, we also collected data from both groups, about serum total proteins, serum albumin, vitamin B12, serum iron, ferritin, hemoglobin, hematocrit and mean corpuscular volume of erythrocytes (MCV).

We diagnosed MetS using the 5 components described by the International Diabetes Federation (IDF), in $2010 .{ }^{9}$ WC was considered high if $\geq 94 \mathrm{~cm}$ in men and $\geq 80 \mathrm{~cm}$ in women. Patients were classified as having diabetes according to recommendations of the American Diabetes Association (ADA) in $2011 .{ }^{10}$ The specific treatment for hypertension included angiotensin-converting enzyme inhibitors (ACEIs), angiotensin receptor blockers (ARBs), ß-blockers, calcium channel blockers, thiazides, loop or potassium-sparing diuretics.

The LDL-chol was considered high when greater than or equal to $160 \mathrm{mg} / \mathrm{dL}$ according to the reference values of LDL-chol in adults (older than 20 years), recommended by the European Association for Cardiovascular Prevention $\&$ Rehabilitation. ${ }^{11}$

The arthralgia and snoring symptoms were collected from medical records. The WL was evaluated by percentage of excess WL (\%EWL) after treatments for both groups. ${ }^{12}$

Data about clinical nutrition complications after treatments were based on physical examinations and findings of patients during their medical visits.

\section{Statistical analysis}

Statistically, the data were expressed as median. To compare the medians of the numerical variables of both groups $(\mathrm{S}$ and NS groups), before and after their treatments, the Mann-Whitney U test was used to compare the medians of the nu- 
merical variables of both groups ( $\mathrm{S}$ and NS), before and after treatment. The Chi-square test was applied for categorical variables. The Wilcoxon T Test was used to compare the data before and after treatments (surgical and clinical) in each group. All statistical analyses were performed in SPSS version 19.0, $\mathrm{p}<0.05$ was considered statistically significant.

\section{Ethical approval}

This study was approved by the Ethics Committees on Humans and Animals at UFCSPA and at the Hospital Santa Casa de Misericórdia de Porto Alegre, RS, Brazil, in accordance with Resolution 196, 1996 of the National Health Council/Ministry of Health of Brazil.

\section{Results}

The baseline characteristics (before treatments) for $\mathrm{S}$ and NS groups are shown in Table 1, indicating that these patients were properly paired and, for this reason, comparable as to their other characteristics.

According to Table 1, in the baseline period, $\mathrm{S}$ and NS groups differed only regarding WC (S - 119 vs. NS $122.00 \mathrm{~cm} ; \mathrm{p}=0.011)$, fasting glucose $(\mathrm{S}-95 v \mathrm{~s}$. NS -98 $\mathrm{mg} / \mathrm{dL} ; \mathrm{p}=0.005)$, serum levels of LDL-chol (S - $118 v s$. NS - $112 \mathrm{mg} / \mathrm{dL} ; \mathrm{p}=0.021)$, and prevalence of T2DM (S - 14.70 vs. NS - 47.30\%; $\mathrm{p}<0.001)$.

TABLE 1 Baseline characteristics of 258 severe obese patients treated with Roux-en-Y gastric bypass (surgical group) or clinically (nonsurgical).

\begin{tabular}{|c|c|c|c|}
\hline & $\begin{array}{l}\text { Surgical } \\
\text { group } \\
(n=129)\end{array}$ & $\begin{array}{l}\text { Nonsurgi- } \\
\text { cal group } \\
(n=129)\end{array}$ & $\begin{array}{l}\mathrm{P} \\
\text { value }\end{array}$ \\
\hline Age (years) & 44.2 & 42.1 & $0.057(\mathrm{a})$ \\
\hline Female sex (\%) & $110(85.3 \%)$ & $106(82.2 \%)$ & $0.078(b)$ \\
\hline $\mathrm{BMI}\left(\mathrm{Kg} / \mathrm{m}^{2}\right)$ & 42.2 & 41.4 & $0.061(a)$ \\
\hline Waist circumference $(\mathrm{cm})$ & 119 & 122 & $0.011(\mathrm{a})^{*}$ \\
\hline MetS (\%) (i) & $68(52.7 \%)$ & $74(57.4 \%)$ & $0.266(b)$ \\
\hline T2DM (\%) (j) & $19(14.7 \%)$ & $61(47.3 \%)$ & $<0.001(b)^{*}$ \\
\hline $\operatorname{HAS}(\%)(\mathrm{k})$ & $62(48.1 \%)$ & $76(58.9 \%)$ & $0.052(b)$ \\
\hline High total-cholesterol (\%) & $60(46.5 \%)$ & $48(37.2 \%)$ & $0.082(b)$ \\
\hline Low HDL-C (\%) & $54(41.9 \%)$ & $67(51.9 \%)$ & $0.067(b)$ \\
\hline High LDL-C (\%) & $43(34.1 \%)$ & $35(27.3 \%)$ & $0.150(b)$ \\
\hline Hypertriglyceridemia (\%) & $52(40.3 \%)$ & $42(32.6 \%)$ & $0.122(b)$ \\
\hline Arthralgias (\%) & $17(13.2 \%)$ & $28(21.7 \%)$ & $0.050(b)$ \\
\hline Number of drugs used & 4.0 & 3.0 & $0.102(a)$ \\
\hline Number of drugs used & 4.00 & 3.00 & $0.102(a)$ \\
\hline
\end{tabular}

The progression of all parameters evaluated from baseline to an average of 1 year after treatments for $\mathrm{S}$ and NS groups is shown in Figures 1 and 2.

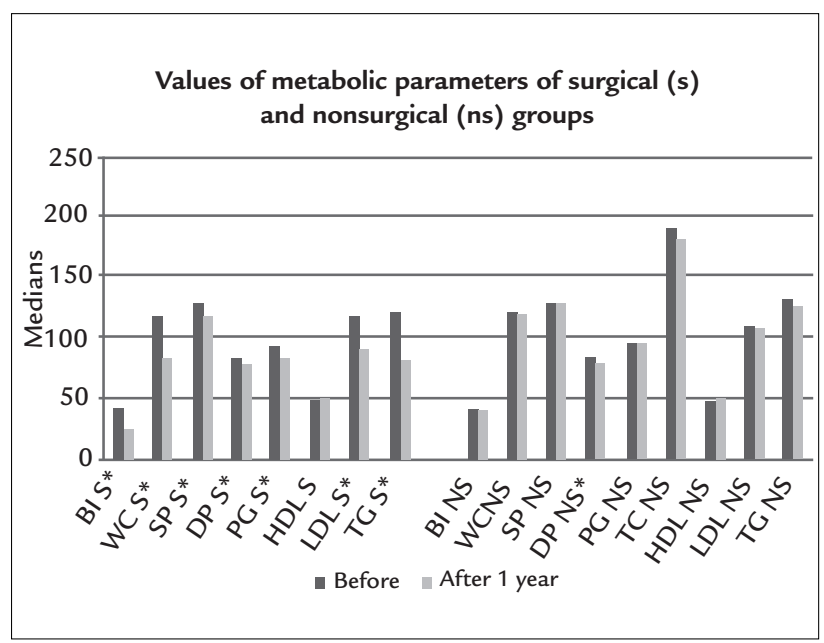

FIGURE 1 Values of metabolic parameters on baseline and 1 year (mean) after interventions in 258 grades 2 and 3 obese patients treated with Roux-en- $Y$ gastric bypass (surgical or $\mathrm{S}$ ) or without surgery (nonsurgical or NS).

*Wilcoxon T test statistically significant $(\mathrm{p}<0.05)$; BI - body mass index $(\mathrm{kg} / \mathrm{m} 2)$; WC waist circumference $(\mathrm{cm})$; $\mathrm{SP}$ - systolic blood pressure $(\mathrm{mmHg})$; DP - diastolic blood pressure $(\mathrm{mmHg})$; PG - plasma glucose (MG/dl); HDL - plasma HDL-cholesterol (mg/dL); LDL- LDL cholesterol (mg/dL); TG - plasma triglycerides ( $\mathrm{mg} / \mathrm{dL}$ ).

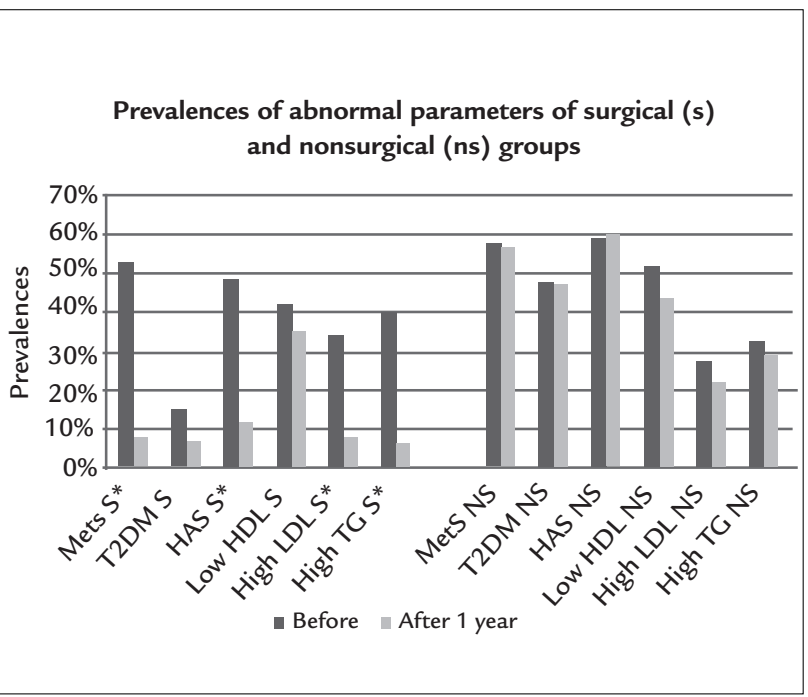

FIGURE 2 Prevalence of abnormal parameters on baseline and 1 year (mean) after interventions in 258 morbidly obese patients treated with Roux-en-Y gastric bypass (surgical or $\mathrm{S}$ ) or without surgery (nonsurgical or NS).

*Wilcoxon T test statistically significant $(p<0.05)$; MetS - metabolic syndrome prevalence; T2DM - type 2 diabetes prevalence; HAS - hypertension prevalence; low HDL - low HDL prevalence; high LDL prevalence; high TG - high triglycerides prevalence. 


\section{Surgical group (SG)}

A more specific analysis of the parameters that significantly differ in $\mathrm{S}$ group in Figures 1 and 2 with a $\mathrm{p}<0.001$, from baseline, is described below.

Mean BMI decreased 38.11\% (from 42.2 to $26.1 \mathrm{Kg}$ / $\mathrm{m}^{2}$ ) and it represented a significant \%EWL of $90.7 \%$. As expected, the waist circumference also decreased (28.6\%; from 119 to $85 \mathrm{~cm}$ ). This group of patients (treated with RYGB) also had a drop in fasting plasma glucose (from 95 to $85 \mathrm{mg} / \mathrm{dL}$ ). They were changed to a smaller number of drugs used to treat hyperglycemia (from 1.0 to 0.0 ).

SBP decreased 7.7\% (from 130 to $120 \mathrm{mmHg}$ ) while DBP decreased by $5.9 \%$ (from 85 to $80 \mathrm{mmHg}$ ); the prevalence of hypertension was reduced (from $48.1 \%$, at baseline, to $11.6 \%$, after RYGB); the number of drugs used for treating hypertension also decreased (from 1.0 to 0.0 ). Together, these results mean that hypertension did improve.

RYGB also determined a drop of $32.5 \%$ in serum Triglycerides (TG) (from 123 to $83 \mathrm{mg} / \mathrm{dL}$ ) and an 84.6\% decrease in the prevalence of hypertriglyceridemia, differing significantly from baseline (from 52 patients - 40.30\% to 8 patients $-20.6 \%$ ). The number of drugs used to treat hypertriglyceridemia was also significantly reduced (from 1.00 to 0.00 ) and, as occurred for hypertension these results define improvement of this dyslipidemia.

The prevalence of patients with MetS decreased 85.2\% (from 68 patients $-52.7 \%$ to 10 patients $-7.8 \%$ ), which means that 58 patients did not meet the criteria for being classified as having MetS. In this study, we consider that a resolution of the MetS occurred.

After RYGB, the serum values of LDL-Chol decreased 21.9\% (from 118.1 to $92.2 \mathrm{mg} / \mathrm{dL}$ ) and the prevalence of high LDL-Chol was reduced by $77.1 \%$, differing significantly from baseline (from 43 patients $-34.1 \%$ to 10 patients $-7.8 \%$ ). The number of drugs used for treating LDLhypercholesterolemia was also significantly reduced in the $S$ group (from 1.0 to 0.0 ). Together, these results could also define another parameter, LDL-hypercholesterolemia, that was improved by the surgical treatment.

The serum levels of HDL-Chol were similar when baseline values were compared with the values obtained on average 12 months after RYGB (50.0 to $52.00 \mathrm{mg} / \mathrm{dL}$ change; $p=0.129$ ). Also, the prevalence of patients with a low serum HDL-cholesterol did not differ significantly from baseline to an average of twelve months period after interventions ( 41.9 to $34.9 \%, \mathrm{p}=0.250$ ).

Prevalence of the symptom of snoring was also evaluated: it decreased $94.8 \%$ (from 20 patients $-15.5 \%$ to 1 patient $-0.8 \%$ ). The $\mathrm{S}$ group showed a significant reduction of $83.3 \%$ in the number of drugs used for the treat- ment of co-morbidities, differing significantly from baseline (from 4.0 to 1.0 ).

With a significant decrease, we had also the variable symptoms of arthralgia, which were frequent among patients at baseline (13.2\%). After RYGB, the prevalence of this symptom decreased by $59 \%$, differing significantly from baseline (from 17 patients - 13.2\% to 7 patients $5.40 \% ; \mathrm{p}=0.032$ ). The number of drugs used to treat the symptoms of arthralgia was also significantly reduced in the $\mathrm{S}$ group compared to the baseline after RYGB (from 1.00 to 0.00$)$.

\section{Nonsurgical group (NSG)}

In the NS group, the mean BMI did not significantly change (from 41.4 to $41.4 \mathrm{Kg} / \mathrm{m}^{2} ; \mathrm{p}=0.497$ ) nor did waist circumference (from 122.0 to $121.0 \mathrm{~cm} ; \mathrm{p}=0.314$ ). Other parameters that did not significantly differ from baseline on an average of 1 year with the NST were the SBP, the prevalence of patients with hypertension, the number of drugs used to treat hypertension, fasting plasma glucose, the number of drugs used to treat hyperglycemia, serum levels of LDL-Chol, number of drugs used for treating LDL-hypercholesterolemia, prevalence of patients with high TG values, number of drugs used to treat hypertriglyceridemia, low HDL-Chol, prevalence of MetS, prevalence of arthralgia and prevalence of patients with the symptom of snoring ( $p>0.05)$.

The DBP of the NS group significantly decreased 5.9\% from baseline after an average of 1 year of treatment (from 85 to $80.00 \mathrm{mmHg}$; $\mathrm{p}=0.031$ ) and the serum levels of TG of twelve months of clinical treatment were $4.1 \%$ inferior to baseline (it went from 134.0 to $128.00 \mathrm{mg} / \mathrm{dL} ; \mathrm{p}=0.047$ ). The number of drugs used to treat the symptoms of arthralgia was significantly increased in the NS group (from 0.0 to $1.0 ; \mathrm{p}=0.023$ ) and an increase of $33.3 \%$ occurred in the number of drugs used for treating co-morbidities (from 3.0 to $4.0 ; \mathrm{p}=0.027$ ).

\section{Surgical compared with nonsurgical groups}

There was no difference between groups for DBP after their respective treatments ( 80 vs. $80 \mathrm{mmHg}$; $\mathrm{p}=0.960$ ) and for prevalence of patients with low HDL-Chol after interventions (respectively 45 patients $-34.9 \%$ vs. 56 patients $-43.4 \%$; $p=0.161$ ).

Moreover, significant differences were observed between the two groups for BMI (respectively $26.10 v$ s. 41.40 $\mathrm{Kg} / \mathrm{m}^{2} ; \mathrm{p}<0.001$ ); \% EWL (respectively $\mathrm{S}-90.70 \%$ vs. NS 3.05\%; $\mathrm{p}<0.001$ ); WC (respectively 85.0 vs. $121.0 \mathrm{~cm}$; $\mathrm{P}<0.001$ ); SBP (respectively 120 vs. $130 \mathrm{mmHg} \mathrm{p}=0.048$ ); prevalence of hypertension (respectively, 15 patients - 11.6\% 
vs. 77 patients - 59.7\%; $\mathrm{p}<0.001)$; number of drugs used to treat hypertension $(0.0 v s .1 .0 ; \mathrm{p}<0.001)$; values of fasting glycemia (respectively, 85.0 vs. $97.0 \mathrm{mg} / \mathrm{dL} ; \mathrm{p}<0.001$ ); number of drugs used to treat hyperglycemia ( $0.0 \mathrm{vs} .1 .0$; $\mathrm{p}<0.001)$ ); and for LDL-Chol (10 - 7.8\% vs. 28 patients 21.7\%; $\mathrm{p}=0.002)$; the number of drugs used for treating LDL-hypercholesterolemia between groups (0.0 vs. 1.0; $\mathrm{p}<0.001$ ); serum TG (83 vs. $128 \mathrm{mg} / \mathrm{dL}$; $\mathrm{p}<0.001$ ); prevalence of hypertriglyceridemia (in the S group - $8-20.6 \%$ vs. the NS group - 37 patients $-28.7 \%$; $\mathrm{p}<0.001$ ); number of drugs used to treat hypertriglyceridemia $(\mathrm{p}<0.001)$; prevalence of MetS (respectively, $10-7.80 \% v s .73$ patients $-56.60 \%$; $\mathrm{p}$ 0.001); symptoms of arthralgia (respectively, $7-5.4 \%$ vs. 32 patients - 24.8\%; $<<0.001$ ); prevalence of the symptom of snoring (respectively, 1 patient $-0.8 \% v$ s. 17 patients - 13.2\%; $\mathrm{p}<0.001)$; number of drugs used for treating co-morbidities (respectively, 1.00 vs. 4.00; $\mathrm{p}<0.001$ ).

With respect to clinical nutritional complications, on an average of 12 months of interventions, it was observed, that the $\mathrm{S}$ and NS groups differed significantly in serum levels of total proteins, which were lower in the S group $(\mathrm{S}-6.8 v$ s. NS $-7.55 \mathrm{~g} / \mathrm{dL} ; \mathrm{p}=0.047)$, serum vitamin $\mathrm{B} 12$, which was also lower in the S group (S - $326 v$ s. NS - 420 $\mathrm{pg} / \mathrm{mL} ; \mathrm{p}=0.035$ ), and on incidence of deficiency of proteins, vitamin B12 and iron, which occurred only in the $\mathrm{S}$ group after RYGB (protein deficiency: $\mathrm{S}-8.5 \%$ vs. NS $0 \%$ patients, $\mathrm{p}=0.011$; vitamin $\mathrm{B} 12$ deficiency: $\mathrm{S} 8.5 \%$ s. NS - $0 \%$ patients, $p=0.011$ and, iron deficiency anemia: $S$ $-6.2 \% v s$. NS $-0 \%$ patients; $\mathrm{p}=0.005$ ).

\section{Discussion}

The results of a retrospective study, which, after an average of one year, evaluated the outcomes of two different treatments offered for treating grades 2 and 3 obese patients in the Brazilian Multidisciplinary Center were compared in the present study. The two treatment options were: 1 . RYGB, performed by trained surgeons plus clinical therapy with patients being evaluated before and after surgery by a multidisciplinary specialized team and 2 . NST alone. It was shown that the surgical procedure was a more effective therapy for obtaining weight loss. As it is well known that this kind of surgery decreases the ingestion of calories and the volume of food consumed, ${ }^{13}$ it was possible to evaluate the effects of this decrease together with the surgical procedure upon several symptoms, metabolic parameters and risk factors for cardiovascular disease.

On average 1 year after RYGB, the present study showed a significant percentage of EWL of $90.70 \%$ for the S group, contrasting with that observed for the NS group that showed a \%EWL of 3.05\%. These data show that on average 1 year after ST, clear benefits of RYGB over the body weight are obtained and it could represent a decrease in many complications related to SO. According to other studies, the \%EWL due to bariatric surgery tends to reach maximum levels between 12 and 18 months after surgery, and varies with the surgical technique. ${ }^{12,14,15}$

Many studies have shown that after RYGP, significant reductions in prevalence of obesity-related co-morbidities could be observed. ${ }^{15-19}$ In the present study, a significant percentage decrease of $85 \%$ of MetS among patients who underwent RYGB on an average of 12 months from its completion (from 68 patients $-52.70 \%$ to $10 \mathrm{pa}$ tients - 7.80\%; $\mathrm{p}<0.001$ ) was observed, contrasting significantly with the NS group, which did not show any change in this parameter. These results show that, as in the case of body weight, clear benefits of RYGB can be obtained for the components of MetS in SO patients. By persisting for a long time, these results could represent the reduction of complications and of high cardiovascular mortality related to MetS. The result observed for the $S$ group in the present study is more impressive than the one described by Batsis et al., ${ }^{3}$ who showed a $58 \%$ decrease in the prevalence of MetS. In this context, our result for the $\mathrm{S}$ group shows that MetS is a largely reversible phenomenon in patients with SO (NNT of 1.2).

With respect to the effects of RYGB on BP, a significant percentage drop of $76 \%$ in prevalence of hypertension was seen in the present study, with the NS group having no changes in the SBP, and in the prevalence of hypertension after intervention. Again, these results show clear benefits of RYGB on BP of SO patients and particularly on the prevalence of hypertension. Since, for the snoring symptoms, a $95 \%$ significant drop in prevalence was found for the RYGB group, with no change in the NS group, it is possible to speculate that the effects on blood pressure in the $\mathrm{S}$ group would be related to sleep apnea since by improving it using other types of treatment, hypertensive levels of blood pressure can decrease. ${ }^{20}$ In the same context, in the Norwegian study of Hofsø et al, ${ }^{4} 76$ patients underwent RYGB and 63 received an NST for intensive lifestyle modifications: clear benefits of RYGB on decreasing the $\mathrm{BP}$ of the $\mathrm{SO}$ patients were shown. Rossi et al. ${ }^{15}$ also showed a similar decrease of $68.1 \%$ in cases of hypertension after surgery.

Since the number of patients with diabetes mellitus who entered the $S$ group was significantly smaller, compared with the NS group, it is difficult to define the exact efficacy of the surgery over this parameter in the present study, after follow-up. However, since we found a $48 \%$ 
significant reduction in the number of patients with diabetes after the follow-up (19 to 10 patients) this difference should be related to the effect of the surgery. Dan et al., ${ }^{18}$ who presented data about $146 \mathrm{SO}$ patients, showed that of 40 patients (27.4\%) who had T2DM before RYGB, $85 \%$ presented complete resolution of this abnormality 4 years after surgery. In the same context, in the study of Hofsø et al., ${ }^{4}$ a remission of T2DM was shown in $70 \%$ in the $\mathrm{S}$ group, $v s .33 \%$ in the intensive lifestyle modification group. The results of these studies show clear benefits of RYGB on the resolution of T2DM and on the decrease of fasting plasma glucose levels in SO patients. It is speculated that the resolution of T2DM after RYGB is related to increased secretion of GLP-1 hormone after meals. GLP-1 secretion in response to meals increases after RYGB and it stimulates the secretion of insulin in diabetic obese patients. Since Buchwald et al. ${ }^{16}$ demonstrated in a meta-analysis that: 1) $85 \%$ of diabetic patients had improved glycemic control after surgery; 2) more than $75 \%$ of the patients with diabetes submitted to RYGP surgery had complete resolution of the diabetes; and 3) part of these findings are related to an improvement in insulin sensitivity, the same events probably happened with the patients in the present study and the drop of fasting blood glucose was related to it.

The results of the present study also show that, on average 12 months after ST, clear benefits of RYGB are obtained for the lipid profile in SO patients. By persisting over the long-term, these results could represent an important reduction in cardiovascular mortality related to lipid profile abnormalities. Decrease of cardiovascular events reported as outcomes was recently confirmed by Sjöstrom et al. ${ }^{17}$ in $\mathrm{SO}$ patients treated with bariatric surgery. As in the present study, Garcia-Marirrodriga et al., ${ }^{19}$ who evaluated 114 patients before and 6, 12 and 18 months after RYGB, showed a decrease in levels of serum T-Chol, LDL-Chol and triglycerides $(\mathrm{p}<0.0005)$, also showing the clear benefits of RYGB on the lipid profile of SO patients.

In the present study, RYGB was responsible for a significant percentage decline of $22 \%$ of serum LDL-Chol and a significant decrease of $77 \%$ in the number of patients with high LDL-Chol. Serum triglycerides were also affected by the RYGB procedure: a decline of $33 \%$ in its levels occurred and the number of patients with hypertriglyceridemia decreased by $85 \%$ after surgery. In a similar period of observation, the NS group showed no changes in parameters like T-Chol and LDL-Chol, differing significantly from the $\mathrm{S}$ group after the interventions. The clinical treatment led to a significant percentage drop of $4 \%$ in serum levels of triglycerides, but this drop did not contribute to a significant reduction of the prevalence of cases of hypertriglyceridemia in this group $(\mathrm{p}=0.05)$.

Neither twelve months of RYGB nor NST were effective in increasing levels of serum HDL-Chol and in decreasing the number of patients with low HDL-Chol. In contrast to the results presented here, Garcia-Marirrodriga et al. ${ }^{19}$ and Silva et al. ${ }^{21}$ showed an increase in serum HDL-Chol levels after surgery. However, these authors did not describe the physical activities of patients after surgery, and Silva et al. studied a group of 96 patients, including 86 women. The frequency and intensity of physical activities were also not evaluated in the present study. We speculate that the patients in our study did not have an increase in their serum levels of HDL-Chol because the surgery and the NST were not effective in promoting it and due to lack of regular practice of physical exercises by both NS and S groups.

With respect to symptoms of arthralgia, RYGB was responsible for a significant percentage drop of $59 \%$ for this symptom. On the other hand, the NS treatment did not change the prevalence of these symptoms. When the groups were compared after the interventions, a significant difference in the number of patients with arthralgia was observed between groups, showing a clear positive effect with the surgical treatment of SO. For this result, we think that it is possible to speculate that the reason for the decrease of patients complaining about joint pain would be the decrease of the impact of body weight upon bone joints.

There were significant reductions in the number of drugs used to treat hyperglycemia, hypertension, hypercholesterolemia and arthralgia after RYGB in the present study. On the other hand, the NS group showed a significant increase of $33 \%$ in the number of drugs used in the period evaluated. When comparing the groups after interventions, we also found a significant difference in this parameter between groups. These results showed an important reduction in the number of drugs used by patients submitted to the surgery and an increase in use of drugs by those treated clinically and with this result we can speculate that the NS group had an aggravation of the co-morbidities. As in our study, Nguyen et al. ${ }^{22}$ and Snow et al..$^{23}$ also showed a significant decrease (92 and $66.7 \%$, respectively) in the number of drugs used by patients to treat their co-morbidities and associated with that finding, the costs of CT decline after the RYGB.

Potentially, weight loss and gastro-intestinal manipulations such as those performed in RYGB, could provide consequences and/or severe complications for patients, especially considering nutritional aspects. ${ }^{12}$ In this con- 
text, the most frequent complications found 1 year after RYGB were the protein-energy deficiency and vitamin deficiencies, especially the vitamin B12 deficiency. Rawlins et al. ${ }^{24}$ in a recent study, showed an incidence of protein and vitamin deficiencies of $21 \%$ in patients evaluated on average 1 year after RYGB. Contrasting with these results, our study showed an incidence of only $8.5 \%$ of protein and vitamin B12 deficiencies and $6.2 \%$ of iron deficiency anemia among patients who underwent the RYGB.

Since the present study compares the data obtained for several co-morbidities, related to obesity grades 2 and 3 after RYGB, with the data obtained with a clinical treatment and the results favor the surgical treatment as being more effective in a 1-year period, we think we need more publications comparing these two situations, in a real life situation, with longer follow-up.

All the data found in the present study suggest that RYGB, due to its effect of decreasing the number of symptoms related to several co-morbidities of SO, could, with less use of drugs, improve quality of life and probably decrease costs and mortality of these patients.

A randomized clinical trial recently published ${ }^{25}$ suggests that RYGB is effective to treat obesity and decreases the cardiovascular risk factors related to it. The present study presents data that coincides with this suggestion and also compares the efficacy of RYGB and that of clinical treatment. In the south of Brazil, the results also clearly favor the surgical approach.

\section{Conclusion}

In a real life Brazilian Multidisciplinary Center for treatment of severe obesity, RYGB, compared to a nonsurgical treatment, in an average period of one year, is a more effective therapy to achieve weight loss, to revert or decrease all the components of MetS, and also to reduce the number of drugs used to treat co-morbidities and their symptoms.

\section{Resumo}

Resolução da síndrome metabólica pela gastroplastia Roux-em-Y em um mundo real: estudo caso-controle.

Objetivo: o objetivo deste estudo foi avaliar a eficácia do bypass gástrico em Y de Roux (BGYR), em comparação a um tratamento não cirúrgico (grupo NC), como instrumento para induzir remissão da síndrome metabólica (SMet) em pacientes com obesidade graus 2 e $3(\mathrm{Ob} 2,3)$.

Métodos: duzentos e cinquenta e oito pacientes com Ob2,3 foram selecionados para análise retrospectiva em estudos de caso e controle. SMet foi definida como descrita pela Federação Internacional de Diabetes. Dos pacientes, 129 foram submetidos ao BGYR (grupo C), e 129 formaram o grupo NC.

Resultados: antes dos tratamentos, os grupos C e NC não diferiram em IMC, idade, sexo feminino e prevalência de SMet $(p>0,05)$. No grupo C, houve uma redução no IMC de 38,1\% ( $\mathrm{p}<0,001)$, circunferência da cintura de $28,6 \%(\mathrm{p}<0,001)$, glicemia de jejum de 10,5\% ( $\mathrm{p}<0,001)$, LDL colesterol sérico de $21,9 \%$ ( $\mathrm{p}<0,001)$ e em $85 \%$ dos casos de SMet $(\mathrm{p}<0,001)$. As diferenças observadas no grupo NC foram uma diminuição nos níveis de triglicérides, de 4,12\% ( $\mathrm{p}=0,047)$, e na pressão arterial diastólica, de $5,9 \%(\mathrm{p}=0,031)$. No grupo NC, $98,6 \%$ dos pacientes persistiram com SMet. O número necessário para tratar (NNT) com a cirurgia, para resolver um caso de SMet, foi de 1,2 (IC 95\%: 1,1 - 1,4).

Conclusão: em um mundo real, no Sul do Brasil, em comparação ao tratamento NS e após 1 ano de observação, BGYR foi altamente eficaz para diminuir a prevalência de SMet.

Palavras-chave: síndrome metabólica X, obesidade, obesidade abdominal, hipertensão, hiperglicemia, hiperlipidemia.

\section{References}

1. Sundström J, Risérus U, Byberg L, Zethelius B, Lithell H, Lind L. Clinical value of the metabolic syndrome for long term prediction of total and cardiovascular mortality: prospective, population based cohort study. BMJ. 2006;332:878-82.

2. Batsis JA, Romero-Corral A, Collazo-Clavell ML, Sarr MG, Somers VK, LopezJimenez F. Effect of bariatric surgery on the metabolic syndrome: A PopulationBased, Long-term Controlled Study. Mayo Clin Proc. 2008;83: 897-906.

3. Sjöström L, Narbro K, Sjöström CD, Karason K, Larsson B, Wedel H et al. Swedish Obese Subjects Study. Effects of bariatric surgery on mortality in Swedish obese subjects. N Engl J Med. 2007;357:741-52.

4. Hofsø D, Nordstrand N, Johnson LK, Karlsen TI, Hager H, Jenssen T, et al. Obesity-related cardiovascular risk factors after weight loss: a clinical trial comparing gastric bypass surgery and intensive lifestyle intervention. Eur J Endocrinol. 2010;163:735-45.

5. Kaul A, Sharma J. Impact of bariatric surgery on comorbidities. Surg Clin North Am 2011;91:1295-312.

6. Salaroli LB, Barbosa GC, Mill JG, Molina MC. Prevalência de síndrome metabólica em estudo de base populacional, Vitória, ES - Brasil. Arq Bras Endocrinol Metab. 2007;51:1143-52.

7. Powell MS, Fernandez AZ Jr. Surgical treatment for morbid obesity: the laparoscopic Roux-en-Y gastric bypass. Surg Clin North Am. 2011;91:1203-24.

8. Benotti P, Wood GC, Winegar DA, Petrick AT, Still CD, Argyropoulos G, Gerhard GS. Risk factors associated with mortality after Roux-en-Y gastric bypass surgery. Ann Surg. 2014;259:123-309.

9. International Diabetes Federation. The IDF Consensus Worldwide Definition of the Metabolic Syndrome, 2010. [cited 2010 apr 1]. Available at: www.idf. org/webdata/docs/IDF-meta-def-final.htm.

10. American Diabetes Association. Summary of revisions of the 2011 Clinical Practice Recommendations. Diabetes Care. 2011;34(1:S3).

11. European Association for Cardiovascular Prevention \& Rehabilitation, Reiner Z, Catapano AL, De Backer G, Graham I, Taskinen MR, Wiklund O, et al. ESC 
Committee for Practice Guidelines (CPG) 2008-2010 and 2010-2012 Committees. ESC/EAS Guidelines for the management of dyslipidaemias: the Task Force for the management of dyslipidaemias of the European Society of Cardiology (ESC) and the European Atherosclerosis Society (EAS). Eur Heart J. 2011;32:1769-818.

12. Montero PN, Stefanidis D, Norton HJ, Gersin K, Kuwada T. Reported excess weight loss after bariatric surgery could vary significantly depending on calculation method: a plea for standardization. Surg Obes Relat Dis 2011;7:531-4.

13. Ziegler O, Sirveaux MA, Brunaud L, Reibel N, Quilliot D. Medical follow up after bariatric surgery: nutritional and drug issues. General recommendations for the prevention and treatment of nutritional deficiencies. Diabetes Metab. 2009;35:544-57.

14. Sjöström L, Lindroos AK, Peltonen M, Torgerson J, Bouchard C, Carlsson B, et al. Swedish Obese Subjects Study Scientific Group. Lifestyle, Diabetes, and Cardiovascular Risk factors 10 years after Bariatric Surgery. N Engl J Med. 2004;351:2683-93.

15. Rossi M, Barretto Fereira da Silva R, Chaves Alcântara G Jr, Regina PF, Martin Bianco Rossi F, et al. Remission of metabolic syndrome: a study of 140 patients six months after Roux-en-Y gastric bypass. Obes Surg. 2008;18:601-6.

16. Buchwald H, Avidor Y, Braunwald E, Jensen MD, Pories W, Fahrbach K, et al. Bariatric surgery. A systematic review and meta-analysis. JAMA. 2004; 292:1724-37.

17. Sjöström L, Peltonen M, Jacobson P, Sjöström CD, Karason K, Wedel H, et al Bariatric surgery and long-term cardiovascular events. JAMA. 2012;307:56-65.
18. Dan D, Harnanan D, Singh Y, Hariharan S, Naraynsingh V, Teelucksingh S. Effects of bariatric surgery on type-2 diabetes mellitus in a Caribbean setting. Int J Surg. 2011;9:386-91.

19. Garcia-Marirrodriga I, Amaya-Romero C, Ruiz-Diaz GP, Férnandez S, BallestaLópez C, Pou JM, et al. Evolution of lipid profiles after bariatric surgery. Obes Surg. 2012;22:609-16.

20. Araghi MH, Chen YF, Jagielski A, Choudhury S, Banerjee D, Hussain S, et al. Effectiveness of lifestyle interventions on obstructive sleep apnea (OSA): systematic review and meta-analysis. Sleep. 2013;36(10):1553-62,1562.

21. Silva MAM, Rivera IR, Gusmão Barbosa EMW, Crispim MAC, Farias GC, Fontan AJA, et al. Frequency of cardiovascular risk factors before and 6 and 12 months after bariatric surgery. Rev Assoc Med Bras. 2013; 59:381-6.

22. Nguyen NT, Varela JE, Sabio A, Naim J, Stamos M, Wilson SE. Reduction in prescription medication costs after laparoscopic gastric bypass. Am Surg. 2006;72:853-56.

23. Snow LL, Weinstein LS, Hannon JK, Lane DR, Ringold FG, Hansen PA, et al. The effect of Roux-en-Y gastric bypass on prescription drug costs. Obes Surg. 2004;14:1031-5.

24. Rawlins ML, Teel D $2^{\text {nd }}$, Hedgcorth K, Maguire JP. Revision of Roux-en-Y gastric bypass to distal bypass for failed weight loss. Surg Obes Relat Dis. 2011;7:45-9.

25. Courcoulas AP, Goodpaster BH, Eagleton JK, Belle SH, Kalarchian MA, Lang W, et al. Surgical vs Medical treatments for type 2 Diabetes Mellitus: A Randomized Clinical Trial. JAMA Surg. 2014;149(7):707-15. 